\title{
Stress Analysis and Optimization of a Flip Chip on Flex Electronic Packaging Method for Functional Electronic Textiles
}

\author{
Menglong $\mathrm{Li}^{\circledR}$, John Tudor, Russel Torah, and Steve Beeby, Senior Member, IEEE
}

\begin{abstract}
A method for packaging integrated circuit silicon die in thin flexible circuits has been investigated that enables circuits to be subsequently integrated within textile yarns. This paper presents an investigation into the required materials and component dimensions in order to maximize the reliability of the packaging method. Two die sizes of $3.5 \mathrm{~mm} \times 8 \mathrm{~mm} \times 0.53 \mathrm{~mm}$ and $2 \mathrm{~mm} \times 2 \mathrm{~mm} \times 0.1 \mathrm{~mm}$ have been simulated and evaluated experimentally under shear load and during bending. The shear and bending experimental results show good agreement with the simulation results and verify the simulated optimal thickness of the adhesive layer. Three underfill adhesives (EP30AO, EP37-3FLF, and Epo-Tek 301 2fl), three highly flexible adhesives (Loctite 4860, Loctite 480, and Loctite 4902), and three substrates (Kapton, Mylar, and PEEK) have been evaluated, and the optimal thickness of each is found. The Kapton substrate, together with the EP37-3FLF adhesive, was identified as the best materials combination with the optimum underfill and substrate thickness identified as $0.05 \mathrm{~mm}$.
\end{abstract}

Index Terms-Bending test, electronic textile, flexible electronic packaging, shear load test, shear strength.

\section{INTRODUCTION}

$\mathbf{I}^{\mathrm{a}}$ $\mathrm{N}$ TODAY'S society, microelectronics technology plays a significant role in people's lives. Electronic textile (e-textiles) technology seeks to further integrate microelectronics technology by combining electronics with textiles to enable a new class of product [1]. An e-textile is a textile with integrated electronic functionality [2], and this can be used in fashion, medical, and military applications [3], [4]. In one approach to realize e-textiles, flip-chip technology is used to mount ultrathin die onto thin flexible plastic film strips that contain patterned conductive interconnects and bond pads. Individual die is located on the strip and the conductive tracks on the plastic substrate link the die together, forming a long, very thin, and flexible circuit. This long thin circuit can then be incorporated into a textile yarn, as shown in Fig. 1, and while

Manuscript received July 11, 2017; revised September 27, 2017 and November 21, 2017; accepted December 3, 2017. This work was supported by the Engineering and Physical Sciences Research Council through the project Novel Manufacturing Methods for Functional Electronic Textiles under Grant EPSRC EP/M015149/1. Recommended for publication by Associate Editor H.-C. Cheng upon evaluation of reviewers' comments. (Corresponding author: Menglong Li.)

The authors are with the Department of Electronic and Computer Science, University of Southampton, Southampton SO17 1BJ, U.K. (e-mail: ml1g12@soton.ac.uk).

Color versions of one or more of the figures in this paper are available online at http://ieeexplore.iee.org.

Digital Object Identifier 10.1109/TCPMT.2017.2780626

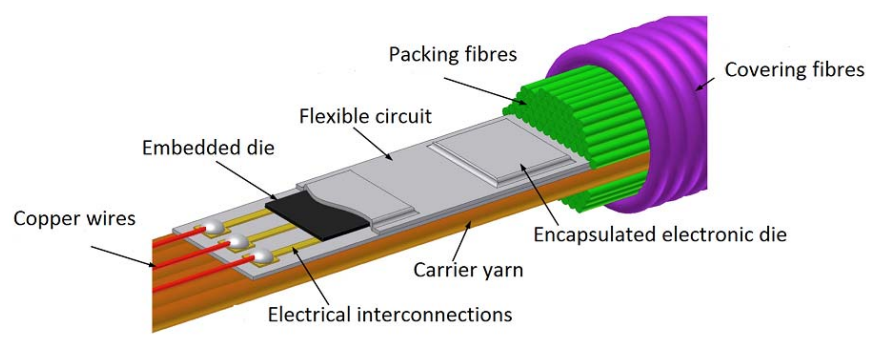

Fig. 1. Schematic of the proposed e-yarn structure and cutaway showing the embedded die.

standard die sizes can be mounted in this way, silicon die that are thin, long, and narrow are preferred. The combination of electronics with textiles requires the use of flexible circuit technology to ensure the textile retains as much as possible their normal physical characteristics and feel [5]. E-textiles will inevitably be used in wearable products and, in such cases, will be subject to human motion and washing, thus making integrated electronic components vulnerable to different forces such as shear and bending loads, which can cause embedded electronic devices to fail.

Reliability and flexibility are two key factors for functional e-textiles [6], and the electronic chip packaging processes described in this paper aim to maximize the reliability of the packaged electronic chip while keeping the package as thin as possible to maximize the flexibility of the overall assembly.

In recent years, significant research has focused on electronic circuit packaging for flexible circuits. The flexibility of electronic circuit does not only involve the substrate, but also concerns the components and interconnections. The review of electronic packaging for system in foil by Koyuncu et al. [7] summarizes the state of the art in this topic. Nowak et al. [8] investigated the mechanical properties of surface-mount device interconnections made on flexible and rigid substrates. In their investigation, the shear strength was measured with a tensile tester on three types of flexible substrate and two types of rigid substrate, and they found the mechanical properties of the joints strongly depend on the configuration of applied materials [8].

The three point bending test [9], [10] is a common method to test the flexibility and reliability of packaged electronic chips. Kim et al. [11] investigated the reliability of chip-in-flex and chip-on-flex packages in a dynamic bending environment 
using anisotropic conductive adhesive. In their investigation, the space under the chip was filled with anisotropic conductive adhesive. In comparison with nonconductive adhesives, anisotropic conductive adhesives provide reduced adhesion and flexibility, and therefore, this approach is more prone to failure [12]. The use of a top covering polyimide film is necessary to achieve suitable reliability under bending [10].

Wire-bonded electronic packages use a nonconductive adhesive to attach the die to the substrate. In typical flip chip packaging, solder is used to make the electrical connection and nonconductive adhesive is applied as an underfill. Underfill is used to: 1) reduce thermal stresses caused by the difference in the coefficient of thermal expansion between the silicon die and the substrate, and 2) increase the reliability of electronic packaging interconnections when mechanical forces and shock loads occur [13]-[16].

In this paper, simulations and experimental evaluation of a flexible electronic packaging method are performed in order to minimize the stress in the adhesive layer of a package designed for e-textiles. Simulations of shear load and bending have been used to investigate the influence of the different adhesives and substrate materials on the packaging stresses, and the simulation results have been experimentally verified. This paper will enable the optimum adhesive and substrate material combination and thicknesses to be identified with confidence by simulation.

Section II introduces the electronic die packaging method as well as the shear load and bending simulations relating to the adhesive and substrate used. The experimental method for testing the packaged electronic chip is presented in Section III. Section IV covers the shear load and bending experimental test results to determine the optimum adhesive thickness and compares these with the simulation results to provide confidence in the simulation method. A new method to determine the adhesion strength of the adhesive is also presented in Section IV. Simulations determining optimum materials and thicknesses of substrate are presented in Section V.

\section{Electronic Packaging Method And Materials}

Fig. 1 shows an idealized schematic in which the electronic die have been completely encapsulated using a thin top plastic layer. This packaging approach is under development within the research group. In order to experimentally validate the simulation techniques being used to optimize this package design, an electronic die on plastic (EDOP) package has been investigated in this paper. The EDOP consists of three layers; the electronic die is mounted on a plastic substrate layer using an adhesive layer, as shown in Fig. 2. Once the simulation processes have been validated, it can be applied with confidence to more complex package shown in Fig. 1.

A 3-D ANSYS finite-element analysis (FEA) model has been used to simulate the EDOP package and to evaluate shear forces and bending stresses. Fig. 2 shows the model of the package. The three boundary conditions applied to the model for the shear force simulation are as shown. An external force is applied to the side surface of the die, the left and right ends of the substrate are fixed in all directions, and

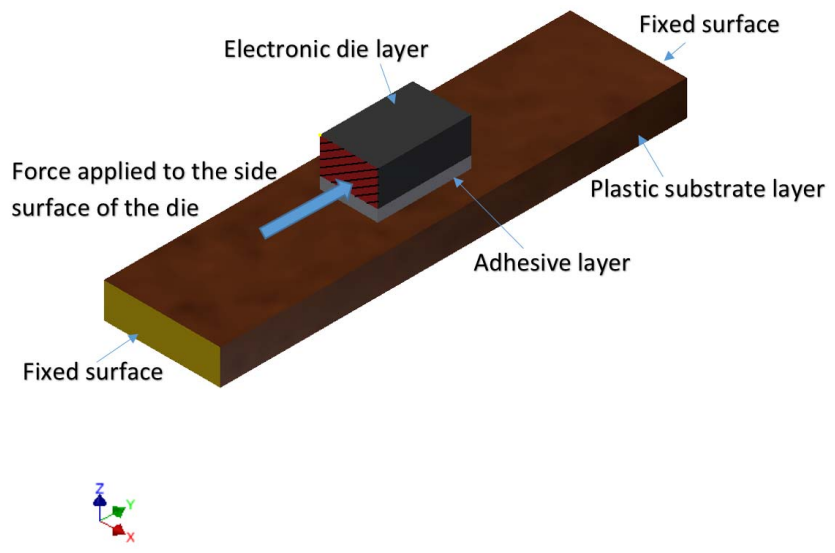

Fig. 2. Shear test model using ANSYS, external force applied to left edge of electronic die.

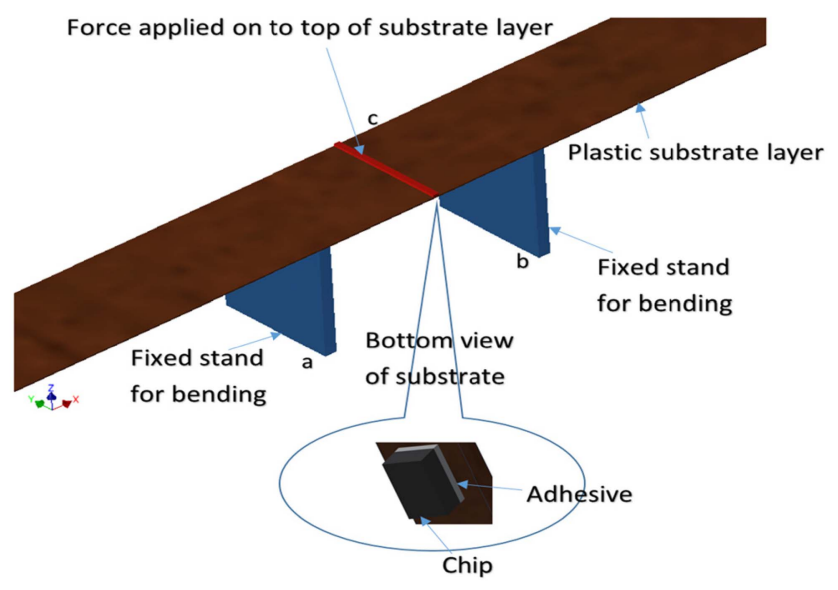

Fig. 3. Three points bending test model using ANSYS.

the top and bottom surfaces of the substrate are fixed in the $z$-direction with the $x$ - and $y$-directions free to move. For the bending simulation, a three-point bending model is used with an external force being applied to the center of the substrate layer on the opposite side to the electronic die. This avoids forces being applied directly to the die, which simplifies the experimental analysis. Fig. 3 shows the model used for the bending FEA simulation. The boundary conditions for the bending simulation are as follows: Parts "a" and "b" are fixed, and therefore, the contact area between substrate and the fixed stand will be fixed. The external force is applied to part "c" in the negative $z$-direction. The contact area of the force applied at point "c" is $0.1 \mathrm{~mm} \times 10 \mathrm{~mm}$, and the contact area for each fixed stand is $1 \mathrm{~mm} \times 10 \mathrm{~mm}$. This replicates the dimension of the fixtures used in the test rig, which uses a probe with a flat tip to control the contact area. The boundary conditions in the simulation match the experimental rigs shown in Fig. 4. The element size was defined by specifying the number of elements along key dimensions of the model. The long edge ( $y$-direction in Fig. 2) and short edge of the die and adhesive were divided into ten and five elements, respectively. The thickness of the die ( $z$-direction) was divided into five elements, while the adhesive 




Rig back plate

(a)

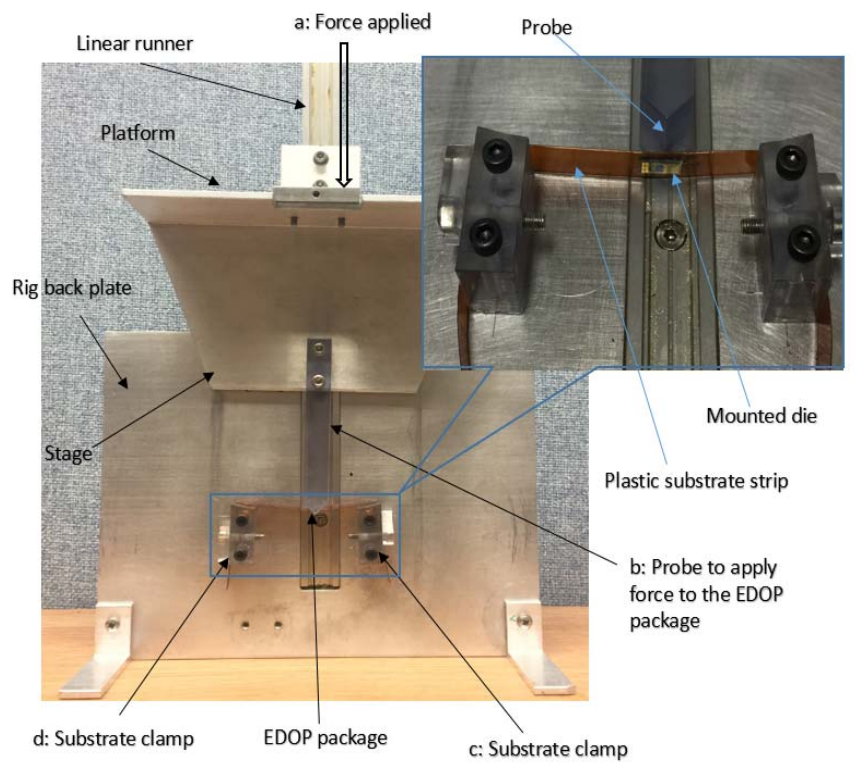

(b)

Fig. 4. Equipment for shear load and bending experiments. (a) EDOP package holder for shear load test. (b) EDOP package holder for bending test.

has three elements through its thickness. The substrate has 20 elements along its length and five elements across its width and through its thickness. The ANSYS Multizone mesh setting was used to perform the mesh operation. To accommodate the different mesh sizes, two contacts were defined between the substrate and the adhesive and also between the adhesive and the die.

In both shear and bending simulations, reliability is determined by observing the stresses in the different layers, with the adhesive material being the most important. From maximum shear stress theory and maximum distortion energy theory [17], the maximum shear stress and von-Mises stress can be determined in order to evaluate the failure of
TABLE I

Adhesive Material Properties From Datasheets [18]-[20]

\begin{tabular}{|l|l|l|l|l|}
\hline $\begin{array}{l}\text { Under-fill } \\
\text { Adhesive }\end{array}$ & $\begin{array}{l}\text { Young's } \\
\text { Modulus } \\
(\mathrm{MPa})\end{array}$ & $\mathrm{CTE}\left(\mathrm{k}^{-1}\right)$ & $\begin{array}{l}\text { Density } \\
\left(\mathrm{gcm}^{-3}\right)\end{array}$ & $\begin{array}{l}\text { Tensile } \\
\text { Strength } \\
(\mathrm{MPa})\end{array}$ \\
\hline EP30AO & 3447 & 0.000025 & 1.06 & 41 \\
\hline $\begin{array}{l}\text { EP37-3FL } \\
\text { F }\end{array}$ & 344 & 0.00009 & 1.05 & 35 \\
\hline $\begin{array}{l}\text { Epo-Tek } \\
3012 \mathrm{fl}\end{array}$ & 3664 & 0.000056 & 1.07 & $\geq 13.7$ \\
\hline $\begin{array}{l}\text { Loctite } \\
\text { 4902 }\end{array}$ & 400 & 0.000425 & 1.06 & 16 \\
\hline $\begin{array}{l}\text { Loctite } \\
4860\end{array}$ & 430 & 0.0001 & 1.07 & $\geq 5$ \\
\hline $\begin{array}{l}\text { Loctite } \\
480\end{array}$ & 2000 & 0.00008 & 1.1 & $\geq 1.8$ \\
\hline
\end{tabular}

TABLE II

Substrate Material Properties From Datasheets [21]-[23]

\begin{tabular}{|l|l|l|l|l|}
\hline $\begin{array}{l}\text { Substrate } \\
\text { Material }\end{array}$ & $\begin{array}{l}\text { Young's } \\
\text { Modulus } \\
(\mathrm{MPa})\end{array}$ & CTE $\left(\mathrm{k}^{-1}\right)$ & $\begin{array}{l}\text { Density } \\
\left(\mathrm{gcm}^{-3}\right)\end{array}$ & $\begin{array}{l}\text { Tensile } \\
\text { Strength } \\
(\mathrm{MPa})\end{array}$ \\
\hline PEEK & 3800 & 0.000026 & 1.32 & 110 \\
\hline Kapton & 2500 & 0.00002 & 1.42 & 231 \\
\hline Mylar & 3100 & 0.000017 & 1.39 & 138 \\
\hline
\end{tabular}

ductile materials. Therefore, the maximum shear stress and von-Mises stress in the adhesive and substrate layer have been analyzed in this paper.

Since circuit flexibility is of key importance in e-textiles, three underfill adhesives (EP30AO, EP37-3FLF, and Epo-Tek $3012 \mathrm{fl}$ ) and three highly flexible adhesives (Loctite 4860, Loctite 480 and Loctite 4902) have been simulated under shear load and bending conditions. The properties of the underfill materials are shown in Table I. Three commonly used flexible substrate materials (Kapton, Mylar, and PEEK) are also compared, and the properties of these are given in Table II.

\section{EXPERIMENTAL METHODS}

To verify the simulation results, shear load [24] and bending experiments have been performed with samples mounted in a way that matches the boundary conditions used in the simulations as closely as possible. Fig. 4(a) shows the rig used for the shear load experiments. The force is applied to a platform located on top of a moveable stage, which is mounted on a linear runner that enables the smooth application of the vertical force to the test EDOP package. The EDOP substrate is held in position by two clamps, which hold the substrate flat against the rig back plate. The edge of the stage is aligned to the side of the die, thereby transferring the force to the 


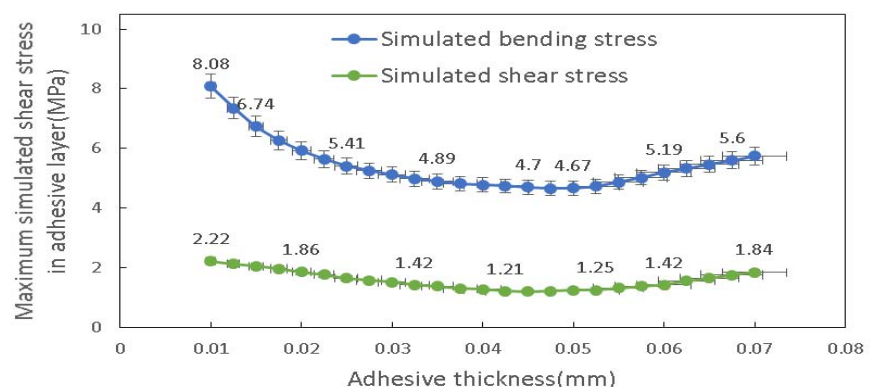

(a)

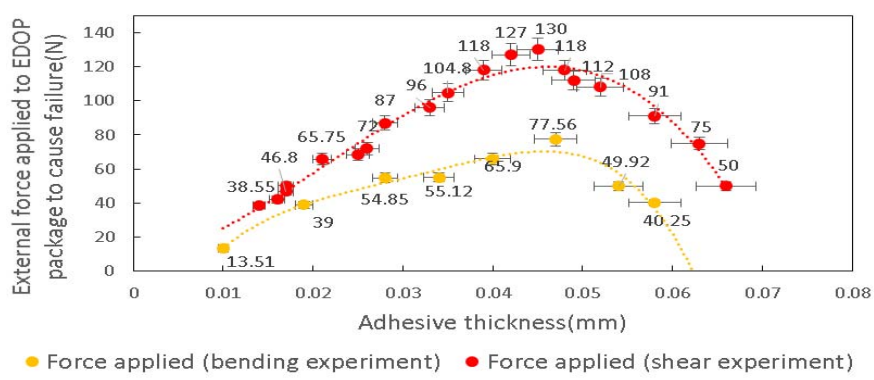

(b)

Fig. 5. Comparison of results for Loctite 4860. (a) Simulated bending and shear stresses versus adhesive thickness (20-N force). (b) External force required to cause failure versus adhesive thickness.

assembled package. This is a block shear test method that replicates the ASTM D4501 testing standard [25].

The bending test rig is shown in Fig. 4(b). Again, the force is applied to the top platform, and this is transferred via a probe to the underside of the substrate, which is clamped at either end. This results in the downward force bending the substrate forming a three-point bending test as shown. In both shear and bending experiments, an Instron Electroplus 1000 mechanical test machine was used to apply the external force to the platform. This has a dynamic load capacity of $\pm 1000 \mathrm{~N}$ and a long-term static load capacity of $\pm 710 \mathrm{~N}$.

\section{VALidATION OF SHEAR LOAD AND BENDING SiMULATIONS}

The shear load and bending experiments have been undertaken to determine the force at which the package fails, as a function of the thickness of the adhesive layer in the EDOP package. For each adhesive, multiple samples (between 6 and 18, one sample at each thickness) were tested, and the external force required to cause the EDOP package to fail is shown in Figs. 5-10 versus adhesive thickness. All six underfill adhesives have been tested in this way with different adhesive thicknesses being achieved by controlling the amount of adhesive dispensed. Each of these adhesives was tested with varying adhesive thickness in the samples, and experimental results were then compared with the simulations. The geometry and material properties affect the coupling of the stress between the films and alter the position of neutral axis of the assembly. For every adhesive, there is an optimum thickness, where the stress in the adhesive is minimized.

The experiment was conducted with a Kapton substrate $180 \mathrm{~mm} \times 10 \mathrm{~mm} \times 0.125 \mathrm{~mm}$, and an $8 \mathrm{~mm} \times 3.5 \mathrm{~mm} \times$ $0.53 \mathrm{~mm}$ silicon die for shear or a smaller $2 \mathrm{~mm} \times 2 \mathrm{~mm} \times$ $0.1 \mathrm{~mm}$ metal die for the bending case. The experimental

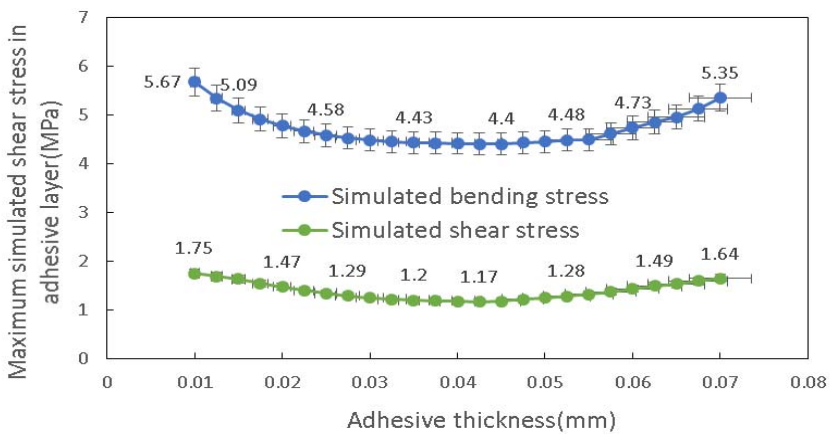

(a)

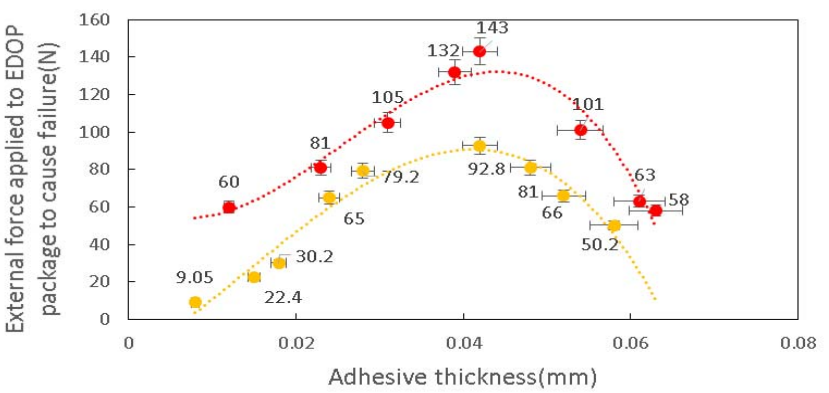

- Force applied (bending experiment) - Force applied (shear experiment)

(b)

Fig. 6. Comparison of results for Loctite 4902. (a) Simulated bending and shear stresses versus adhesive thickness (20-N force). (b) External force required to cause failure versus adhesive thickness.

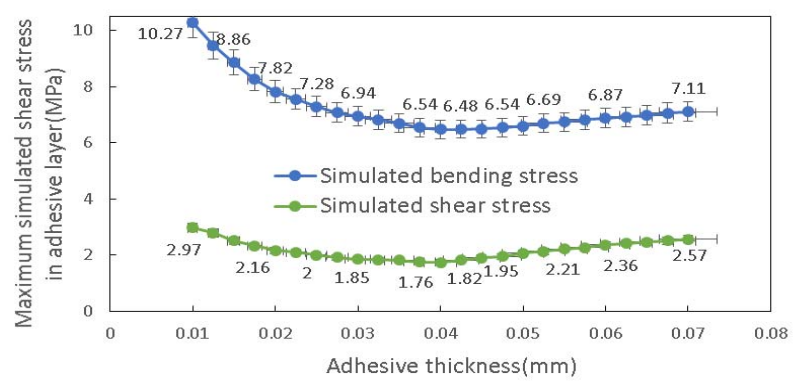

(a)

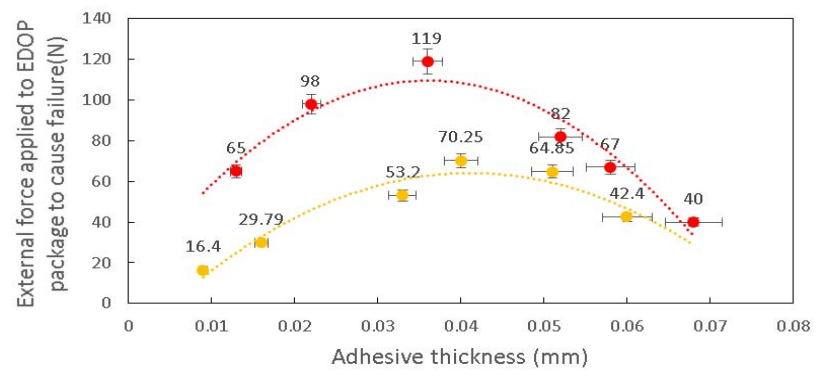

- Force applied (bending experiment) • Force applied (shear experiment)

(b)

Fig. 7. Comparison of results for Loctite 480. (a) Simulated bending and shear stresses versus adhesive thickness (20-N force). (b) External force required to cause failure versus adhesive thickness.

and simulated evaluation of two different die sizes and materials enable a more in-depth comparison between the experimental and simulation results providing greater 


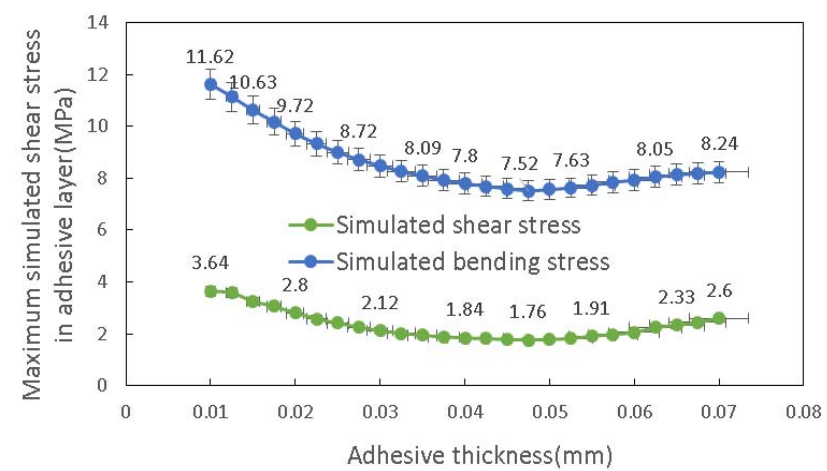

(a)

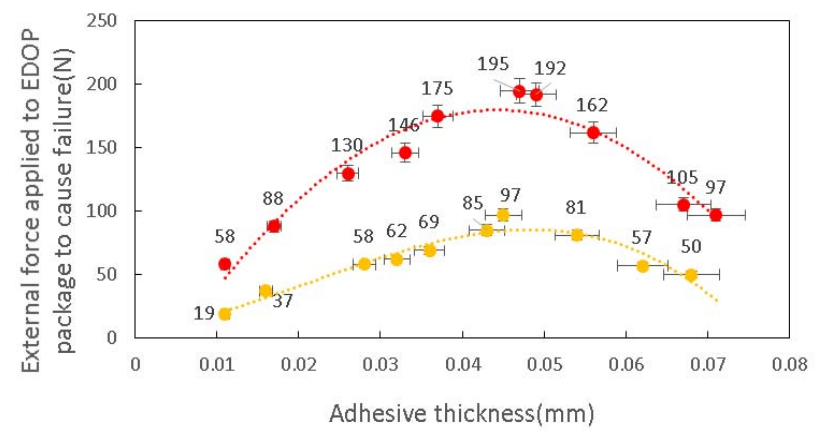

- Force applied (shear experiment) • Force applied (bending experiment)

(b)

Fig. 8. Comparison of results for EP30AO. (a) Simulated bending and shear stresses versus adhesive thickness (20-N force). (b) External force required to cause failure versus adhesive thickness.

confidence in the validation process. In each case, the length and width of the adhesive layer match the die dimension in the EDOP package.

The simulation results in this validation exercise consist of the stress in the adhesive for different thicknesses. At the optimum thickness, the simulation results should indicate minimum stress in the adhesive compared with other thicknesses. For the experimental results, the maximum force required to break the package compared with other thicknesses will indicate the optimum thickness. The validation exercise compares the simulation result with the experimental result. The thicknesses of the adhesive layer are between 0.01 and $0.07 \mathrm{~mm}$, and a $20-\mathrm{N}$ shear load and bending force are applied in the simulations.

Fig. 5 shows the simulation and experimental results for Loctite 4860. The simulation results for shear load and bending are shown in Fig. 5(a), which indicates the optimal thickness of adhesive is between 0.042 to $0.047 \mathrm{~mm}$ in both bending and shear cases. The experimental results are shown in Fig. 5(b), and this indicates that the optimal adhesive thickness of the Loctite 4860 layer in the shear load experiment is in the range from 0.042 to $0.045 \mathrm{~mm}$. For the bending experiment, the optimal thickness is between 0.045 and $0.048 \mathrm{~mm}$. The optimal thickness in both simulation and experimental results closely correlate and the optimum adhesive thickness shows good agreement, which indicates that at that thickness the stress in the adhesive layer is minimized. The fact that the adhesive fails in-elastically and

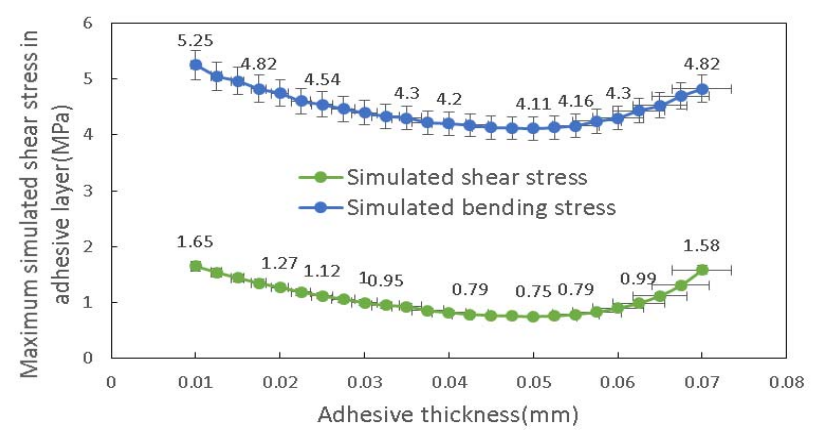

(a)

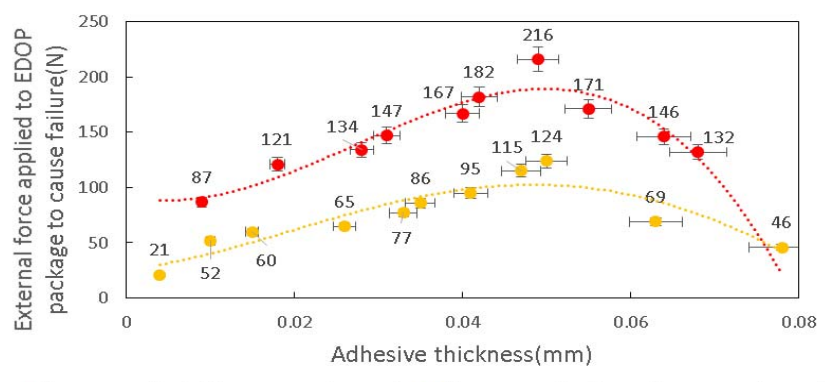

- Force applied (shear experiment) • Force applied(bending experiment)

(b)

Fig. 9. Comparison of results for EP37-3FLF. (a) Simulated bending and shear stresses versus adhesive thickness (20-N force). (b) External force required to cause failure versus adhesive thickness.

TABLE III

Optimum Thickness Results For SiX AdHesive Materials

\begin{tabular}{|l|l|l|}
\hline Adhesive & $\begin{array}{l}\text { Optimum thickness } \\
(\mathrm{mm}) \text { - simulation }\end{array}$ & $\begin{array}{l}\text { Optimal thickness } \\
(\mathrm{mm}) \text { - experiment }\end{array}$ \\
\hline Loctite 4860 & $0.042-0.047$ & $0.042-0.048$ \\
\hline Loctite 4902 & $0.04-0.045$ & 0.042 \\
\hline Loctite 480 & 0.04 & $0.038-0.04$ \\
\hline EP30AO & 0.047 & $0.047-0.049$ \\
\hline EP37-3FLF & 0.05 & $0.048-0.05$ \\
\hline Epo-Tek 301 2fl & $0.045-0.047$ & $0.043-0.045$ \\
\hline
\end{tabular}

the simulation was performed elastically does not affect the agreement between the results-there is clearly an optimum adhesive thickness, where the stress induced in the adhesive by the applied load is minimized. The small error between simulation and experiment is due in part to the tolerance in measuring the adhesive thickness. The thickness of the adhesive layer for each sample was determined by measuring the total thickness of the sample minus the thickness of the substrate and die. All thicknesses were measured using a digital micrometer with a tolerance of $0.002 \mathrm{~mm}$.

Both simulation and experimental results have indicated an optimum thickness exists for all adhesives tested. The comparison between simulation and experiment for Loctite 4902, Loctite 480, EP30AO, EP37-3FLF, and Epo-Tek $3012 \mathrm{fl}$ are shown in Figs. 6-10, respectively. The optimum adhesive thickness for each material is shown in Table III.

In simulation, for the same adhesive thickness, the simulated shear stress in the EP30AO and Epo-Tek $3012 \mathrm{fl}$ adhesives 




(a)

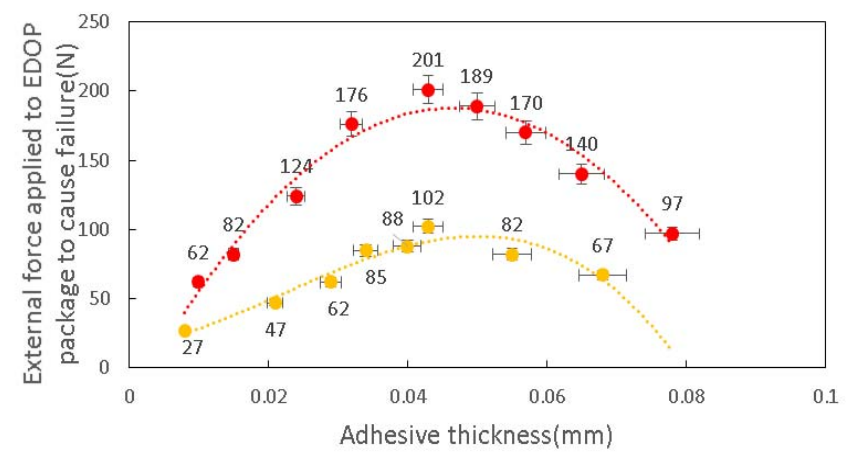

- Force applied (shear experiment) - Force applied (bending experiment)

(b)

Fig. 10. Comparison of results for Epo-Tek 301 2fl. (a) Simulated bending and shear stresses versus adhesive thickness (20-N force). (b) External force required to cause failure versus adhesive thickness.

in shear and bending cases is much higher than for the Loctite 4902. However, in the shear and bending experiments, the force needed to break the EP30AO and Epo-Tek $3012 \mathrm{fl}$ package is much higher than the Loctite 4902 package. This is because the EP30AO and Epo-Tek $3012 \mathrm{fl}$ adhesives have much higher shear strength than Loctite 4902. So for the adhesives with similar shear strength, the smaller shear stress simulated in bending and shear cases shows improved stress performance.

These comparisons show very good correlation between the experimental and simulation results for both shear and bending cases for all materials and dimensions. This validation exercise provides a high level of confidence in the simulation approach, and therefore, this has been used to look further into the design of the EDOP package. These simulations are presented in Section V.

\section{A. Adhesive Strength}

The magnitude of the experimental forces resulting in failure of the Loctite 480, Loctite 4860, Loctite 4902, EP30AO, EP37-3FLF, and Epo-Tek 301 2fl adhesives during the shear test can be applied in the simulations to identify the practical shear strength of each adhesive. To do this, the failure force identified for each sample in the shear load experiment was applied in the simulation along with the adhesive thickness to determine the stress in the adhesive at the point each

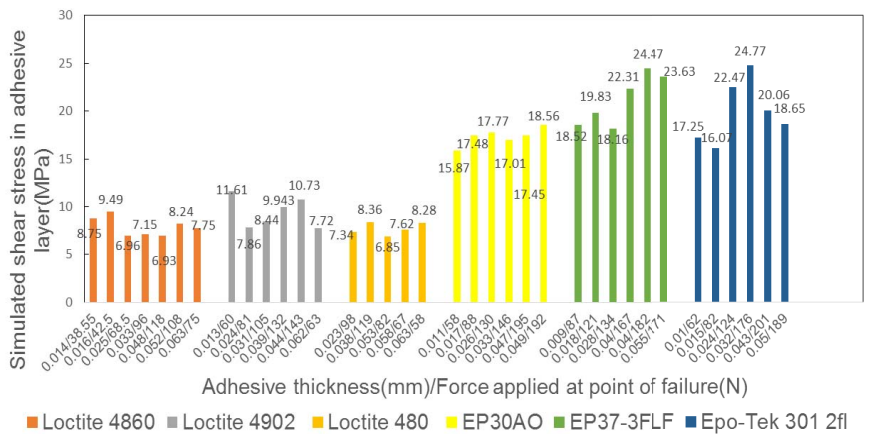

(a)

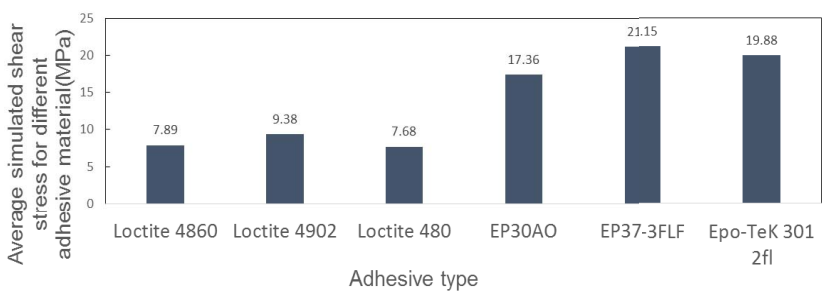

(b)

Fig. 11. (a) Shear strength in different thicknesses of six adhesives in simulation. (b) Shear strength comparison of the six adhesives.

sample failed. The shear strength of the adhesive can be determined by averaging the simulated failure shear stresses from all the samples. The failure origin was found to be the corners of the die close to the location of the applied force. All shear stress values in the simulations are taken from the corners where the external the force was applied. The failure mode in both the shear and bending experiments indicated the adhesive failed at the chip/adhesive interface or within the adhesive itself.

Fig. 11(a) shows how to use this approach to obtain the shear strength the six adhesives. For Loctite 4860, for example, the forces at which the package failed are $38.55,42.5,68.5$, $96,118,108$, and $75 \mathrm{~N}$ for the adhesive at thicknesses of 14 , $16,25,33,48,52$, and $63 \mu \mathrm{m}$, respectively. The simulated shear strength in each instance is $8.75,9.49,6.96,7.15,6.93$, 8.24, and 7.75 MPa, as shown in Fig. 11(a). The average shear strength of Loctite 4860 is $7.89 \mathrm{MPa}$.

This approach has been applied to all the adhesives, and the average shear strength of each adhesive when bonding silicon to Kapton is shown in Fig. 11(b). This simulation result shows that, of the six adhesives, the EP37-3FLF adhesive has the highest practical shear strength.

\section{Shear Load and Bending Simulation Results}

The simulations in this section were used to determine the influence of the adhesive, substrate material, and substrate thicknesses on the stress present in the adhesive during bending and shear loading. This will enable the optimum underfill and substrate material and thicknesses to be determined for each combination of materials. The length and width of the substrate are fixed at $180 \mathrm{~mm} \times 10 \mathrm{~mm}$ for both simulations. A fixed size of $8 \mathrm{~mm} \times 3.5 \mathrm{~mm} \times 0.53 \mathrm{~mm}$ silicon die is used. 


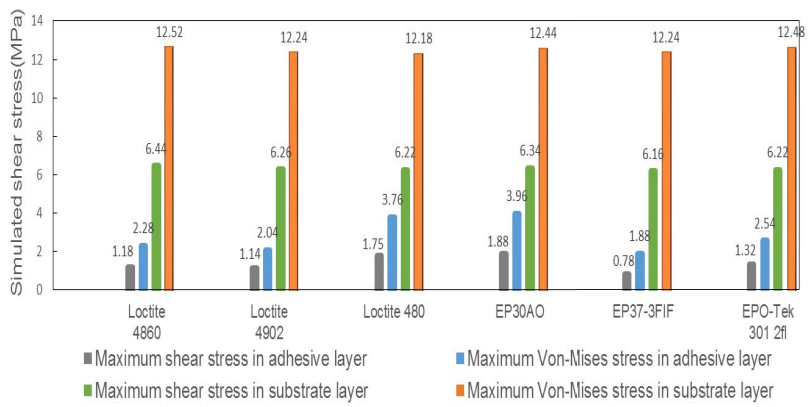

(a)

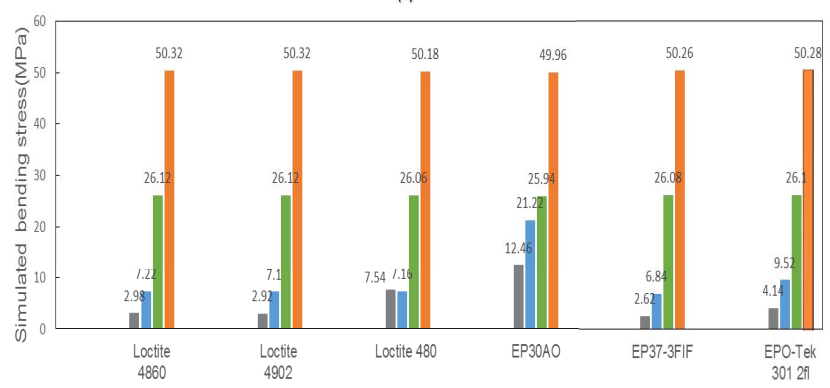

- Maximum shear stress in adhesive layer Maximum Von-Mises stress in adhesive layer

॥aximum shear stress in substrate layer \Maximum Von-Mises stress in substrate laye

Fig. 12. Shear and von-Mises stress simulations to compare adhesives with a Kapton substrate. (a) Shear load model. (b) Bending model.

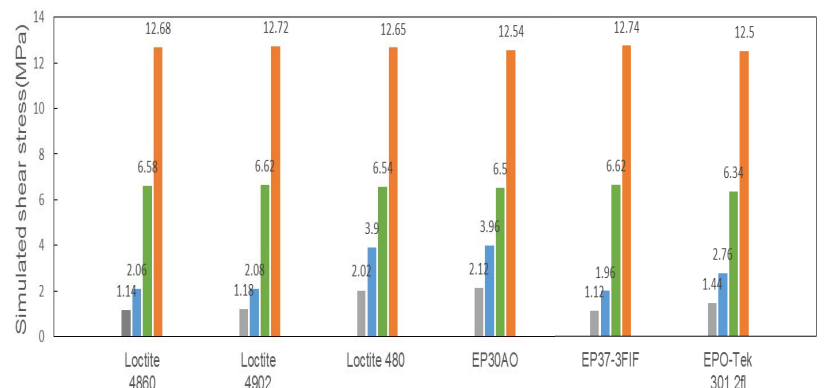

-Maximum shear stress in achesive layer aMaximum Von-Mises stress in achesive layer -Maximum shear stress in substrate layer Maximum Von-Mises stress in substrate layer (a)

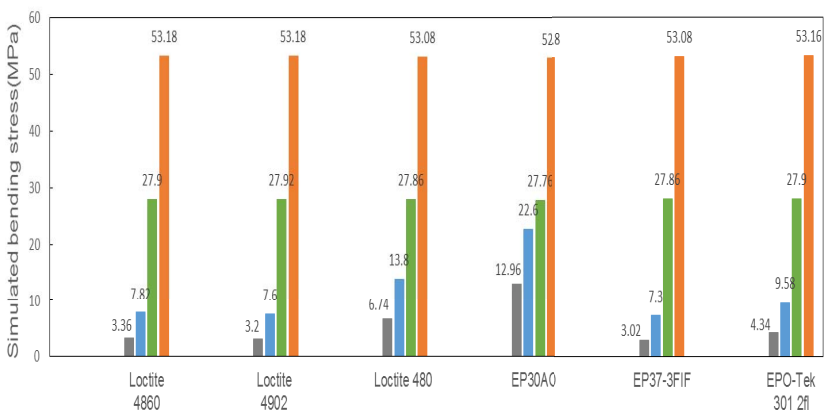

IMaximum shear stress in achesive layer IMaximum Von-Mises stress in achesive layer = Maximum shear stress in substrate layer a Maximum Von-Mises stress in substrate layer

Fig. 13. Shear and von-Mises stress simulations to compare adhesives with a Mylar substrate. (a) Shear load model. (b) Bending model.

\section{A. Effect of Underfill and Substrate Material}

The six underfill adhesives were simulated on each substrate material in turn, and the stress induced in the adhesive and
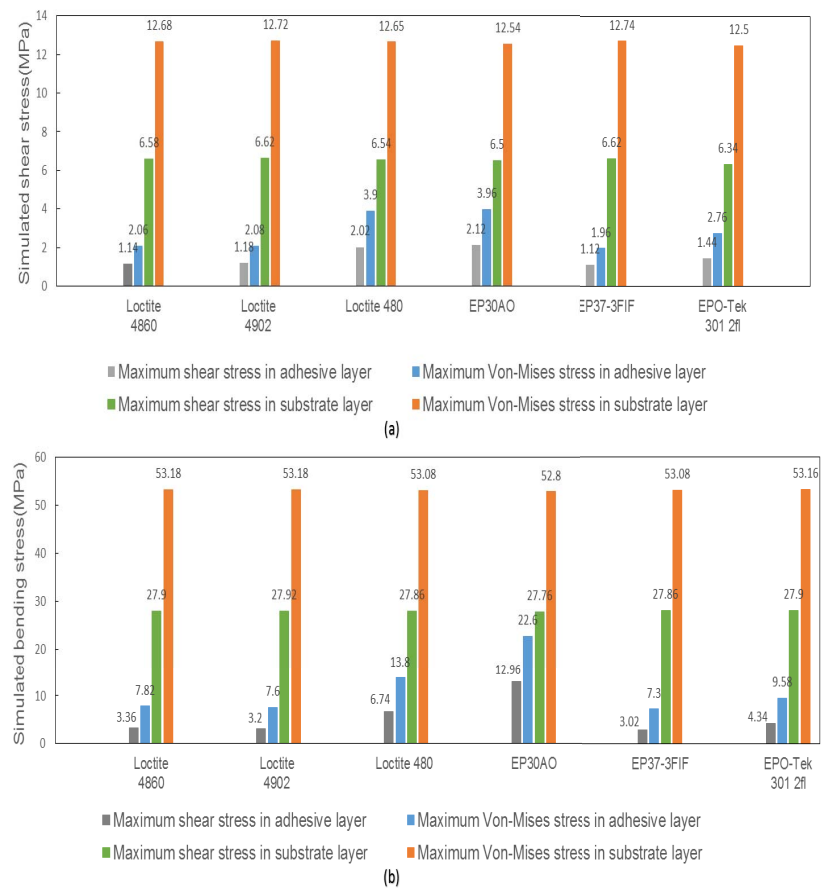

Fig. 14. Shear and von-Mises stress simulations to compare adhesives with a PEEK substrate. (a) Shear load model. (b) Bending model.

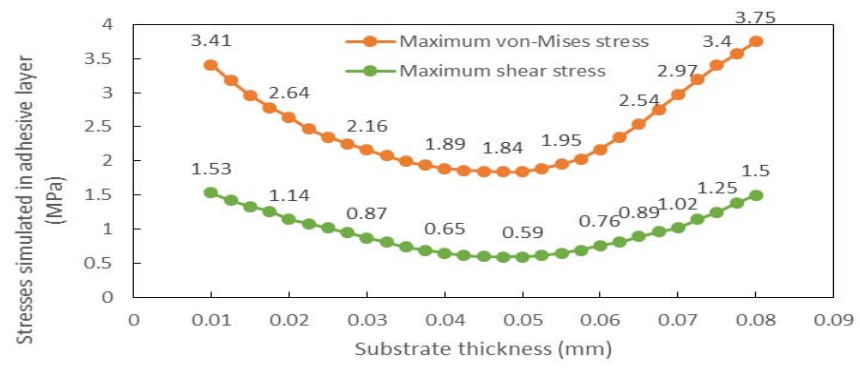

(a)

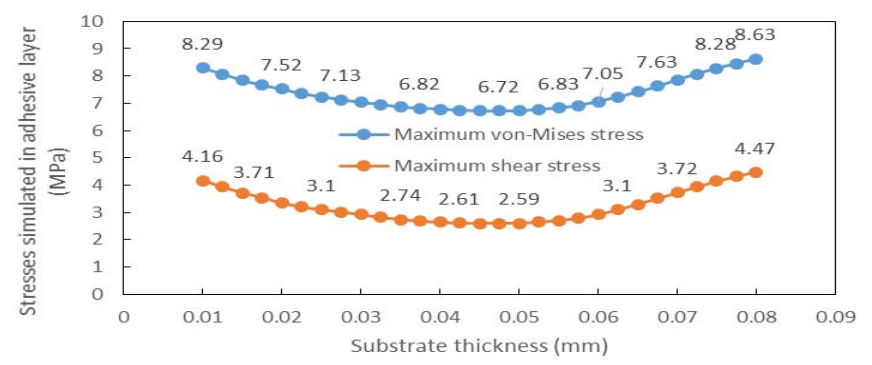

(b)

Fig. 15. Stress simulation $20 \mathrm{~N}$ applied load to determine the optimal thickness of the substrate layer. (a) Shear stress and von-Mises stress against substrate thickness under shear. (b) Shear stress and von-Mises stress against substrate thickness under bending.

substrate layers determined. The best combination of substrate and adhesive materials for the EDOP package is identified by the lowest stress in the adhesive and substrate layers. Figs. 12-14 show the maximum shear and Von-Mises stresses for the Kapton, Mylar, and PEEK substrates, respectively. The thickness of the substrate layer and adhesive layer is fixed at 
0.125 and $0.05 \mathrm{~mm}$, respectively, and the external force applied in the shear load and bending simulation is $20 \mathrm{~N}$.

Of the six underfill adhesives, the EP37-3FIF shows the lowest shear and Von-Mises stress in the adhesive layer under shear load and bending. The shear and Von-Mises stress in the substrate do not change significantly for each underfill material.

With regard to the choice of substrate material, Kapton results in the lowest stress level in the adhesive layer and the substrate and when combined with the EP37-3FIF adhesive. Therefore, this was identified as the best material combination.

\section{B. Effect of Substrate Thickness}

This section presents the investigation into the effect of substrate thickness on the stress induced in the adhesive. The results are shown in Fig. 15. The adhesive used is EP37-3FIF, and the optimum adhesive thickness of $0.05 \mathrm{~mm}$ is taken from the previous results, and the thickness of the Kapton varied between 0.01 and $0.08 \mathrm{~mm}$. The external force applied in the shear load and bending simulations is $20 \mathrm{~N}$. A Kapton thickness of between 0.048 and $0.052 \mathrm{~mm}$ gives the lowest stress in the adhesive, as shown in Fig. 15.

\section{CONCLUSION}

This paper investigated the influence of the adhesive and substrate thickness and material on the stresses in the EDOP package. The different material combinations of the EDOP package of different adhesive materials (EP30AO, EP37-3FLF, Epo-Tek 301 2fl, Loctite 4902, Loctite 4860, and Loctite 480 ) and different substrates (Kapton, Mylar and PEEK) have been compared. A 0.048- to 0.052-mm-thick Kapton substrate and a $0.05-\mathrm{mm}$ EP37-3FIF adhesive layer were identified as the optimum materials and thicknesses for the EDOP package. The simulation results show very good correlation with experimental results that gives a high level of confidence in this simulation approach. The method to determine the practical shear strength presented in Section IV can be used to determine the shear strength for any adhesive when combined with a specific material (such as Kapton or silicon). The simulations can now be used to improve the more complex package shown in Fig. 1, which has top plastic cover film to protect the die.

\section{ACKNOWLEDGMENT}

Data published in this paper are available from the University of Southampton repository at 10.5258/SOTON/D0141.

\section{REFERENCES}

[1] E. Bonderover and S. Wagner, "A woven inverter circuit for e-textile applications," IEEE Electron Device Lett., vol. 25, no. 5, pp. 295-297, May 2004.

[2] R. Bhattacharya, M. M. de Kok, and J. Zhou, "Rechargeable electronic textile battery," Appl. Phys. Lett., vol. 95, no. 22, p. 223305 , 2009.

[3] D. Meoli and T. May-Plumlee, "Interactive electronic textile development: A review of technologies," J. Textile Apparel, Technol. Manag., vol. 2, no. 2, pp. 1-11, 2002.
[4] T. Martin, M. Jones, J. Edmison, T. Sheikh, and Z. Nakad, "Modeling and simulating electronic textile applications," in Proc. ACM SIGPLAN/SIGBED Conf. Lang., Compilers, Tools Embedded Syst., New York, NY, USA, 2004, pp. 10-19.

[5] C. Zysset, T. W. Kinkeldei, N. Munzenrieder, K. Cherenack, and G. Troster, "Integration method for electronics in woven textiles," IEEE Trans. Compon., Packag., Manuf. Technol., vol. 2, no. 7, pp. 1107-1117, Jul. 2012.

[6] R. B. Katragadda and Y. Xu, "A novel intelligent textile technology based on silicon flexible skins," Sens. Actuators A, Phys., vol. 143, no. 1 , pp. 169-174, 2008.

[7] M. Koyuncu, E. Lorenz, and A. Zimmermann, "Advanced interconnection technologies for flexible organic electronic systems," in Handbook of Flexible Organic Electronics: Materials, Manufacturing and Applications. Sawston, U.K.: Woodhead, 2014, pp. 143-169.

[8] D. Nowak, A. Dziedzic, Z. Żaluk, H. Roguszczak, and M. Węglarski, "Mechanical properties of SMD interconnections on flexible and rigid substrates," Soldering Surf. Mount Technol., vol. 28, no. 1, pp. 27-32, 2016.

[9] Y.-S. Lai, T. H. Wang, H.-H. Tsai, and M.-H. R. Jen, "Cyclic bending reliability of wafer-level chip-scale packages," Microelectron. Rel., vol. 47, no. 1, pp. 111-117, 2007.

[10] I. Kim and S. B. Lee, "Reliability and failure analysis of leadfree solder joints for PBGA package under a cyclic bending load," IEEE Trans. Compon. Packag. Technol., vol. 31, no. 2, pp. 478-484, Jun. 2008

[11] J.-H. Kim, T.-I. Lee, J.-W. Shin, T.-S. Kim, and K.-W. Paik, "Ultra-thin chip-in-flex (CIF) technology using anisotropic conductive films (ACFs) for wearable electronics applications," in Proc. 65th Electron. Compon. Technol. Conf., May 2015, pp. 714-718.

[12] R. C. Benson, D. Farrar, and J. A. Miragliotta, "Polymer adhesives and encapsulants for microelectronic applications," Johns Hopkins Appl. Tech. Dig., vol. 28, no. 1, pp. 58-71, 2008.

[13] Y. C. Chan, M. O. Alam, K. C. Hung, H. Lu, and C. Bailey, "Effect of underfill entrapment on the reliability of flip-chip solder joint," J. Electron. Packag., vol. 126, no. 4, pp. 541-545, 2004.

[14] J. B. Nysaether, Z. Lai, and J. Liu, "Thermal cycling lifetime of flip chip on board circuits with solder bumps and isotropically conductive adhesive joints," IEEE Trans. Adv. Packag., vol. 23, no. 4, pp. 743-749, Nov. 2000.

[15] K. I. Loh, S. Chang, and E. S. Ibe, "Underfill encapsulants and edgebond adhesive for enhanceing of board level reliability," in Proc. SMTA Int Conf., 2013, pp. 1-5.

[16] J. W. Wan, W. J. Zhang, and D. J. Bergstrom, "Recent advances in modeling the underfill process in flip-chip packaging," Microelectron. J., vol. 38, no. 1, pp. 67-75, 2007.

[17] V. B. Bhandari, "Design against static load," in Design of Machine Elements. New Delhi, India: McGraw-Hill, 2010, pp. 107-110.

[18] Masterbond. EP30AO Product Description, EP37-3FLF Product Description. Accessed: Mar. 2, 2017. [Online]. Available: https://www.masterbond.com/products/list-all-two-component-epoxysystems

[19] E. Technology. EPO-TEK 301-2FL. Accessed: May 13, 2016. [Online]. Available: http://www.epotek.com/site/component/products/products. $\mathrm{html}$ ?title $=\&$ search $=0 \&$ sign $=0 \&$ value $=$

[20] Henkel. Loctite 4902, Loctite 480 and Loctite 4860. Accessed: Apr. 26, 2015. [Online]. Available: http://www.na.henkel-adhesives.com/ product-search-1554.htm?pageIndex $=9 \&$ primaryFacet $=00000 X Y Z 01 \#$

[21] Boedeker. PEEK (PolyEtherEtherKetone) Specifications. Accessed: Oct. 18, 2015. [Online]. Available: http://www.boedeker.com/peek p.htm?utm_source=google\&utm_medium $=$ cpc\&utm_campaign $=$ PEEK\%20-\%20International\&utm_content=peek\%20sheet\&utm_term $=$ \%2Bpeek\%20\%2Bsheet\&gclid=CO_ks6CM3tMCFaK37QodSXsP8Q

[22] DuPont. Kapton HN General-Purpose Polyimide Film. Accessed: Oct. 23, 2015. [Online]. http://www.dupont.com/products-andservices/membranes-films/polyimide-films/brands/kapton-polyimidefilm/products/kapton-hn.html

[23] UKI. Mylar Polyester Film Datasheet. Accessed: Mar. 28, 2017. [Online]. Available: http://usa.dupontteijinfilms.com/wp-content/ uploads/2017/01/Mylar_Electrical_Properties.pdf

[24] A. Singh, D. A. Horsley, M. B. Cohn, A. P. Pisano, and R. T. Howe, "Batch transfer of microstructures using flip-chip solder bonding," J. Microelectromech. Syst., vol. 8, no. 1, pp. 27-33, Mar. 1999.

[25] Standard Test Method for Shear Strength of Adhesive Bonds Between Rigid Substrates by the Block-Shear Method, Standard ASTM D4501, 2014. [Online]. Available: www.astm.org/Standards/D4501.htm 


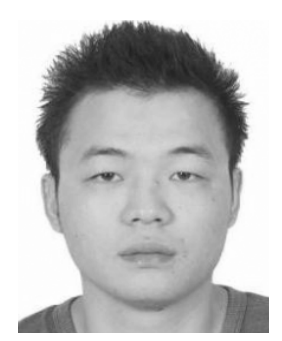

Menglong Li received the B.Eng. degree in electronic engineering from the University of Southampton, Southampton, U.K., in 2014, where he is currently pursuing the Ph.D. degree with the Department of Electronic and Computer Science.

His current research interests include flexible electronic packaging method, screen printing, dispenser printing, ultrathin chip fabrication, and reliability test of electronic packaging.

John Tudor received the Ph.D. degree in physics from the University of Surrey, Guildford, U.K.

$\mathrm{He}$ is currently a Principal Research Fellow with the University of Southampton, Southampton, U.K. His current research interests include smart fabrics, screen printing, dispenser printing, microelectromechanical systems, microsystems, energy harvesting, sensors, resonant sensors, inkjet printing, and wireless sensors.
Russel Torah received the Ph.D. degree in electronics from the University of Southampton, Southampton, U.K., in 2004.

He is currently a Senior Research Fellow with the University of Southampton. His current research interests include smart fabric development, energy harvesting, sensors, and transducers.

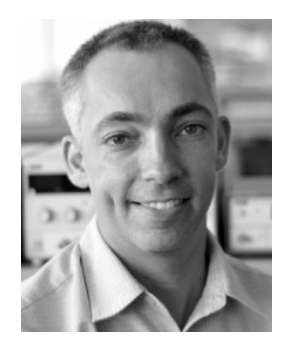

Steve Beeby (SM'11) received the Ph.D. degree from the University of Southampton, Southampton, U.K., in 1998.

He is currently a Professor with the Department of Electronics and Computer Science, University of Southampton, where he is the Head of the Electronics and Electrical Engineering Group. He has authored or coauthored 114 refereed journal papers and letters, 140 refereed conference papers, 5 review papers, 5 books, and 11 chapters within books, and he has an $h$-index of 43 . He holds ten patents and has co-founded three companies. His current research interests include e-textiles, energy harvesting, and printed low-temperature active materials for flexible and textile substrates. 